\title{
EL USO DE LAS TECNOLOGÍAS DE LA INFORMACIÓN Y COMUNICACIÓN (TIC) POR LOS MIGRANTES SUDAMERICANOS EN LA ARGENTINA Y LA CONFORMACIÓN DE REDES SOCIALES TRANSNACIONALES
}

Cecilia Melella ${ }^{1}$

\begin{abstract}
El uso de las TIC implica un punto de inflexión para los procesos migratorios tradicionales al posibilitar que el migrante desarraigado se transforme en migrante conectado. Las TIC, y en especial Internet, generan un universo frondoso de posibilidades de conexión y comunicación con los países de origen, con la diáspora y con la colectividad en destino que modifica la construcción de la subjetividad migrante y conforman redes sociales transnacionales. En este artículo, nos proyectamos dar cuenta de la importancia de las TIC, más allá de considerarse herramientas y artefactos culturales de la esfera pública, como dispositivos a los que los migrantes recurren para construir un espacio referencial que admite la conservación de vínculos asiduos con las redes sociales en origen y se mitiga o reconfigura la nostalgia de la ausencia y/o del retorno. Nos proponemos, a través de la realización de entrevistas en profundidad y cuestionarios, estudiar los medios y las tecnologías más utilizadas por los individuos pertenecientes a estos grupos para comunicarse con sus redes sociales en origen, las causas de su elección, los usos más frecuentes y las temáticas que despiertan su interés a la hora de recurrir a estos dispositivos tecnológicos.
\end{abstract}

Palabras clave: migraciones sudamericanas, tecnologías de la información y comunicación (TIC), apropiación y uso, migrante conectado, redes sociales transnacionales.

\section{1 - Introducción}

A lo largo de la historia moderna, los migrantes han utilizado tecnologías tradicionales como el correo postal, el telégrafo o el teléfono para contactarse con su lugar de origen. Las nuevas tecnologías de la información y de la comunicación

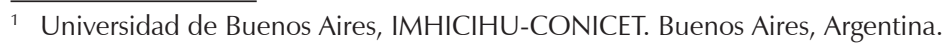


(TIC), como productos de un proceso complejo y en constante cambio, se han diversificado en nuevas formas como teléfonos celulares y la red Internet dentro de la cual circulan heterogéneos programas de conexión y comunicación como Facebook, Skype, Whatsapp o incluso Twitter². De tal forma, podemos afirmar que las TIC, como nuevos medios y/o artefactos culturales ${ }^{3}$, potencian y cambian los usos y las mediaciones ${ }^{4}$ que las personas les daban a los artefactos pasados ${ }^{5}$ tomando cada vez mayor trascendencia en las distintas esferas de la vida de los hombres y, en los últimos años, han adquirido una envergadura relevante dentro del campo de los estudios sobre los procesos migratorios transnacionales ${ }^{6}$. En un contexto migratorio, la apropiación y el uso de estas tecnologías han permitido mantener más fluidos los círculos afectivos y relativizar la ruptura nostálgica causada por la lejanía7. También han potenciado la conformación de una doble presencia: la física (o real) y la imaginada (o virtual), la de estar aquí y allá pudiendo participar en la comunidad de origen y en la de destino ${ }^{8}$. En este sentido, las TIC implican un punto de inflexión para los procesos comunicacionales en contextos migratorios al potenciar el pasaje de un migrante desarraigado a un migrante más conectado. Dentro de la esfera subjetiva del migrante, las TIC influyen directamente en la conformación y expresión de las narrativas migratorias (historias de vida, narrativas mediáticas, acceso a la información y construcción de la biografía) tanto en el plano individual como colectivo. Así, los migrantes globalizados, además de conservar vidas territorializadas, mantienen conexiones virtuales que les permiten construir su subjetividad y desarrollar su biografía ${ }^{9}$. En consecuencia, este artículo se propone

2 MELELLA, Cecilia. Apropiación y uso de las tecnologías de la información y de la comunicación (TIC) en la prensa migrante de las colectividades andinas en la Argentina; IDEM. Migración y Tecnologías de la Información y de la Comunicación (TIC). La presencia de los periódicos de migrantes en Internet y los desafíos del análisis de las redes sociales virtuales. IDEM. Migración y tecnologías de la información y la comunicación (TIC). De la prensa gráfica a las redes virtuales como espacios de construcción identitaria de los migrantes de países andinos en la Argentina; IDEM. MIGRANTES.COM. Las asociaciones de migrantes andinos en la Web; IDEM. Migración y TIC: Identidades andinas en Facebook.

3 HINE, Christine. Etnografía virtual.

4 MARTín-BARBERO, Jesús. De los medios a las mediaciones: Comunicación, cultura y hegemonía. CASTELLS, Manuel. La Era de la Información: Vol. I: La Sociedad Red.

6 CASTELO STARKOFF, Paula, RAMIREZ, Jacques. Migración ecuatoriana y uso de nuevas tecnologías de Información y Comunicación; CARDON, Dominique. La innovación por el uso. Palabras en juego. Enfoques Multiculturales sobre las Sociedades de la Información; PEÑARANDA CÓLERA, María Cristina. ¿Tecnologías que acercan distancias? Sobre los 'claroscuros' del estudios de la(s) tecnología(s) en los procesos migratorios transnacionales; BRUNEAU, Michel. Diaspora, transnational spaces and communities; KISSAU, Kathrin, HUNGER, Uwe. The internet as a means of studying transnationalism and diaspora; GONZÁLVEZ TORRALBO, Herminia. Comunicación y contacto transnacional: El uso de las nuevas tecnologías de información y comunicación en la constitución de redes migratorias; MELELLA, Cecilia, PERRET, Gimena. Tecnologías de la Información y de la Comunicación y estudios migratorios. Reflexiones sobre un campo de estudios incipiente; entre otros.

7 MEjÍA ESTEVEZ, Silvia. Transnacionalismo a la ecuatoriana: migración, nostalgia y nuevas tecnologías.

${ }^{8}$ DIMINESCU, Dana. El migrante conectado. Por un manifiesto epistemológico.

9 CASTEllO, STARKOFF, Paula, BURBANO, Mauricio. Migración ecuatoriana y uso de Nuevas 
reflexionar sobre el uso de las TIC por parte de los migrantes sudamericanos en la Argentina -desde un plano subjetivo- como punto de encuentro entre las historias de vida individuales y las prácticas colectivas en contextos globalizados. Seleccionamos a los migrantes sudamericanos, en primer lugar, porque dichas colectividades componen la migración más numerosa en la Argentina. Según el Censo Nacional de Población, Hogares y Vivienda de la Argentina de 2010 residen en el país 550.713 paraguayos, 345.272 bolivianos, 191.147 chilenos, 157.514 peruanos, 116.592 uruguayos, 41.330 brasileños, 17.576 colombianos y 4.820 ecuatorianos, entre los grupos más destacados. En segundo lugar, porque entendemos que resultan significativos los estudios sobre la vinculación entre el uso de las TIC y las migraciones desde un contexto sur-sur en un entorno académico mundial donde se manifiestan más frecuentes los estudios de la migración sur-norte. En síntesis, nos interesa indagar sobre los medios y las tecnologías más usadas por los individuos pertenecientes a estos grupos para comunicarse con sus redes sociales en origen, las causas de su elección, los usos más frecuentes y las temáticas que despiertan su interés a la hora de recurrir a estos dispositivos tecnológicos.

\section{2 - El abordaje teórico-metodológico}

Entendemos la apropiación de las TIC como una operación práctica e interpretativa en la cual se ponen en juego imaginarios, representaciones y prácticas socioculturales ${ }^{10}$. El uso de la tecnología no implica la mera relación con el objeto, sino que involucra la correspondencia con un universo de representaciones culturales (técnica) con las que esa tecnología se articula. En consecuencia, partimos de la caracterización de Internet desde una doble dimensión. Por un lado, como artefacto cultural, una tecnología, una herramienta que adquiere significados culturales y usos diferentes en relación a los contextos donde es utilizada. Por otro lado, como un lugar, un espacio donde se gesta una cultura ${ }^{11}$.

Las TIC se conforman como herramientas y como espacios de despliegue de narrativas migratorias, pues, por un lado, los migrantes construyen su biografía y conforman su subjetividad atravesados por estas tecnologías de la presencia. Por otra parte, las TIC, específicamente Internet, permite la conexión, la exhibición y puesta en común de historias compartidas. Es decir, no sólo los migrantes narran su vida a través de los distintos soportes tecnológicos (páginas Web, Facebook, Instagram, entre otros) sino que el uso de estos dispositivos contribuye a delinear su biografía cotidiana a través de la posibilidad de contacto en tiempo real con su círculo social y con su universo simbólico del origen.

Tecnologías de Información y Comunicación; GÓMEZ-ESCALONILLA MORENO, Gloria, CAMPOS ZABALA, Marina. Espacios de solidaridad en Internet: Webs para inmigrantes.

${ }^{10}$ WINOCUR, Rosalía. La apropiación de la computadora e Internet en los sectores populares urbanos.

${ }^{11}$ HINE, op. cit. 
De este modo, las TIC como objeto de estudio resultan un fenómeno comunicacional que presenta múltiples dimensiones y requiere para su abordaje la combinación de saberes diversos como el análisis de discurso, la sociosemiótica, la antropología, la sociología y hasta la ciencia política. A partir de una metodología cualitativa, proponemos la utilización del método biográfico basado en la técnica de la entrevista en profundidad ${ }^{12}$. Esta técnica admite situarse entre lo individual y lo colectivo, lo público y lo privado, en "un espacio entre que clausura la antinomia, revelando la imbricación profunda entre individuo y sociedad"13. Asimismo, recurrimos a la realización de cuestionarios abiertos sobre la temática. Realizamos una totalidad de 80 entrevistas y cuestionarios entre los años 2011 y 2015 considerando una muestra heterogénea de migrantes procedentes de países sudamericanos que vivieron en la Argentina y cuyas experiencias migratorias estuvieron atravesadas por el uso de las $\mathrm{TIC}^{14}$. Por otra parte, realizamos una base de datos sobre las páginas Web y cuentas de Facebook de las principales colectividades sudamericanas en la Argentina ${ }^{15}$.

\section{3 - Antecedentes. TIC y migraciones}

La vinculación entre las TIC y los procesos migratorios compone una línea de investigación novedosa en la Argentina y en el resto del mundo ${ }^{16}$. Un referente resulta la investigación de Dana Diminescu ${ }^{17}$ quien ha postulado el concepto de migrante conectado ${ }^{18}$. Como sostiene Diminescu ${ }^{19}$, las TIC han modificado las

\footnotetext{
12 VASILACHIS DE GIALDINO, Irene. Estrategias cualitativas de investigación.

${ }^{13}$ ARFUCH, Leonor. El espacio biográfico: Dilemas de la subjetividad contemporánea.

${ }^{14}$ No tuvimos en cuenta la distinción por clase social, género, nacionalidad o edad entre otras características, pues el objetivo de este artículo consiste en realizar un estudio sobre el uso general que los migrantes le dan a las TIC.

${ }^{15}$ Una parte de la base de datos fue utilizada en mi tesis doctoral Migración y tecnologías de la información y la comunicación (TIC). De la prensa gráfica a las redes virtuales como espacios de construcción identitaria de los migrantes de países andinos en la Argentina. Tesis de doctorado no publicada. Universidad de Buenos Aires, Facultad de Ciencias Sociales, Argentina. En esta investigación se trabajó sobre la construcción identitaria de los migrantes de países andinos en la Argentina (bolivianos, peruanos, colombianos y ecuatorianos) en los medios de comunicación comunitarios y en las redes virtuales. Se utilizó una metodología cualitativa basada en el análisis de discurso y contenido de una selección de páginas Web y cuentas de Facebook de periódicos y asociaciones de cada colectivo. Asimismo, se conformó una base de datos de páginas Web y cuentas de Facebook de las asociaciones y periódicos de cada grupo y se trabajó con los programas informáticos Navicrawler y Gephi que permitieron graficar la conectividad virtual de cada uno de éstos. Paralelamente se realizaron entrevistas en profundidad y cuestionarios abiertos a migrantes de las comunidades seleccionadas.

${ }^{16}$ MELELLA, PERRET, op. cit.

${ }^{17}$ DIMINESCU, El migrante conectado..., op. cit.; IDEM. E-Disporas Atlas. Exploration and Cartography of Disaporas in Digital Networks.

${ }^{18}$ El migrante conectado se define como aquel que por medio del uso de las TIC puede desarrollar actividades, fomentar redes (reales o virtuales) y estilos de vida que le permiten mantenerse conectado con su país de origen, de destino y con la diáspora (IDEM, El migrante conectado..., op. cit.; IDEM, E-Disporas Atlas..., op. cit.).

${ }^{19}$ IDEM, El migrante conectado..., op. cit.
} 
formas de migrar tradicionales caracterizadas por la pérdida del vínculo con el país de origen, sus redes sociales y familiares. Las TIC transforman el desarraigo en conexión y promueven el contacto diario y el mantenimiento de vínculos. Estas tecnologías, a su vez, potencian la construcción de redes transnacionales ${ }^{20}$. Por su parte, Arjun Appadurai ${ }^{21}$ ha señalado que las TIC y las migraciones constituyen ejemplos de confluencia de las prácticas de un vivir trasnacional.

Otros estudios como los de Georgiou ${ }^{22}$ y Goube $^{23}$ han analizado la relación entre los procesos migratorios y las TIC desde su producción mediática. Las producciones de sitios Web de asociaciones de migrantes y las redes sociales virtuales promovidas por la Web $2.0^{24}$ como Facebook y Twitter o por la Web 3.0 ${ }^{25}$ han facilitado el contacto directo entre personas en distintos lugares del mundo, creando relaciones no sólo entre los países de origen y destino, sino conectado a las diásporas mundiales ${ }^{26}$. Particularmente, en el tema que proponemos, España constituye un ejemplo paradigmático para comparar con el caso argentino. Ese país resulta representativo en este tipo de estudios debido, entre otras causas, a su inmigración reciente de países latinoamericanos y africanos. Los principales análisis realizados a lo largo de la última década ${ }^{27}$ coinciden en el incremento de la presencia de las colectividades en la Web ${ }^{28}$. Los inmigrantes no cortan afectiva ni comunicativamente con su tierra natal, por lo cual, la posibilidad de leer (en tiempo real) la prensa de sus países de origen o la prensa de cada colectividad emitida en la sociedad receptora conectan aún más el aquí y el allá.

Como hemos mencionado, en la Argentina son escasos los estudios que han abordado esta problemática. Entre ellos es destacable la investigación de Gimena Perret $^{29}$ quien ha estudiado las TIC como herramientas para la participación

${ }^{20}$ PORTES, Alejandro. Convergencias teóricas y evidencias empíricas en el estudio del transnacionalismo de los inmigrantes.

${ }^{21}$ APPADURAI, Arjun. La modernidad desbordada: Dimensiones culturales de la globalización.

${ }^{22}$ GEORGIOU, Myria. Diasporic Media Across Europe: Multicultural Societies and the UniversalismParticularism Continuum.

${ }^{23}$ GOUBE, Josephine. When immigration meets innovation: immigrants 2.0.

${ }^{24}$ Conjunto de servicios que permiten confeccionar, modificar, almacenar, implantar y compartir información y conservarla en la red. Uno de los casos emblemáticos es Facebook.

${ }^{25}$ La Web 3.0, también denominada como Web semántica, tiene como objetivo actuar como una guía "inteligente" que provea información personalizada para cada usuario.

${ }^{26}$ MELELLA, Migración y tecnologías de la información..., op. cit.

${ }^{27}$ GONZÁLEZ CORTÉS, María Eugenia, BARRANQUERO CARRETERO, Alejandro. Empleo y usos de internet en las comunidades imaginadas: la red como herramienta neutralizadora de la distancia; CACHÓN RODRÍGUEZ, Lorenzo. La España inmigrante. Marco discriminatorio, mercado de trabajo y políticas de integración; GÓMEZ-ESCALONILLA MORENO, CAMPOS ZABALA, op. cit., entre otros.

${ }^{28}$ No obstante, durante el año 2008, como producto de la crisis económica, política y social que sufrió España junto a otros países europeos, se ha evidenciado una reducción de los medios de comunicación de migrantes consecuencia de la reducción de los subsidios, privados y estatales, recibidos, así como del retorno de gran parte de la población inmigrada.

${ }^{29}$ PERRET, Gimena. Nuevas tecnologías de la información, formas de organización migrante y lucha por derechos políticos de la migración chilena en Argentina. 
política de los inmigrantes chilenos. Por su parte, mis investigaciones se han centrado en el análisis de la construcción de identidad de las colectividades de países andinos en la Argentina a partir del uso de las $\mathrm{TIC}^{30}$. Por último, resulta relevante para nuestro artículo el trabajo de $\operatorname{Arfuch}^{31}$, quien, desde el método biográfico en las ciencias sociales, reflexiona sobre la construcción de la identidad y de una memoria biográfica en los emigrantes argentinos a Italia a finales de los años noventa y su relación con los medios de comunicación y el uso de la tecnología. La autora rescata la voz del otro en el relato de la experiencia de la migración a partir de entrevistas en profundidad con el objetivo de descubrir el despliegue del espacio biográfico.

\section{4 - Apropiación y uso de las TIC. Una aproximación desde el plano subjetivo en contextos migratorios}

Como señalamos en la introducción, las TIC atraviesan los procesos migratorios en un plano individual, en la construcción de subjetividades, así como en las prácticas sociales colectivas. En investigaciones realizadas previamente $^{32}$ se demostró que las TIC jugaban un papel significativo en la conformación del universo social, cultural y, específicamente, comunicacional de los migrantes al proporcionar otro espacio expresivo para la conformación identitaria y el despliegue de un nuevo tipo de tecno-sociabilidad migratoria ${ }^{33}$. Los colectivos migratorios se apropiaron de diversos saberes, como aquellos que tenían que ver con el universo del periodismo, para fundar periódicos y revistas de sus colectividades que luego han pasado al ciberespacio como páginas Web de periódicos o cuentas de Facebook de asociaciones. Estos medios de comunicación virtuales se despliegan como espacios para la conformación de identidades, funcionan como medios de información y de opinión, como lazos de cohesión comunitaria y como recursos de visibilidad en la sociedad receptora ${ }^{34}$. Sin embargo, además del uso colectivo dado por las asociaciones o los medios de comunicación comunitarios, nos preguntamos por la importancia que adquieren estas tecnologías en la configuración de la historia de vida de cada migrante.

El Censo Nacional de Población, Hogares y Vivienda de la Argentina de 2010 indica las siguientes cifras para el uso de la computadora por parte de los colectivos de migrantes sudamericanos: 150.756 paraguayos, 100.026 bolivianos, 63.313 chilenos, 94.347 peruanos, 59.831 uruguayos, 18.159 brasileños, 14.177 colombianos y 3.803 ecuatorianos. Cada uno de estos grupos ha utilizado los

\footnotetext{
${ }^{30}$ MELELLA, Migración y tecnologías de la información..., op. cit.

31 ARFUCH, op. cit.

${ }^{32}$ MELELLA, Migración y tecnologías de la información..., op. cit.

33 PEÑARANDA CÓlERA, op. cit.

${ }^{34}$ MELELLA, La prensa migrante..., op. cit.; IDEM. Apropiación y uso..., op. cit.; IDEM. Migración y Tecnologías..., op. cit.; IDEM. Migración y tecnologías..., op. cit.
} 
dispositivos de forma heterogénea. Por ejemplo, con respecto a la presencia en Internet, las colectividades con más años de radicación en la Argentina como los bolivianos, paraguayos y chilenos no manifiestan poseer una mayor cantidad de sitios Web y páginas de Facebook de asociaciones, instituciones y medios de comunicación que los grupos migratorios más recientemente asentados como los ecuatorianos y colombianos. En el año 2013 hallamos 3 páginas Web de ecuatorianos y 4 cuentas de Facebook, 8 páginas de colombianos y 41 de cuentas de Facebook frente a 48 páginas Web de asociaciones y 96 cuentas de Facebook de los bolivianos. Los peruanos poseían 55 páginas Web y más de una centena de cuentas de Facebook. En el caso de los chilenos entre 10 y 16 cuentas de Facebook, mientras que las páginas Web tuvieron mayor desarrollo entre los años 2000 y 2005, registrando al menos 5 sitios Web de centros y asociaciones de chilenos en Argentina ${ }^{35}$.

\section{Figura 1 - Tipos de tecnologías de la información y de la comunicación utilizados por los migrantes sudamericanos en Argentina}

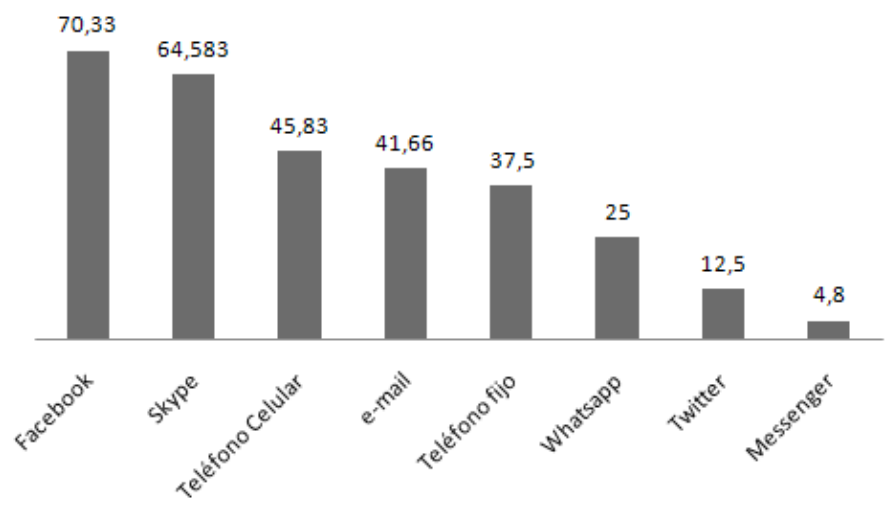

Fuente: Elaboración personal sobre la base de datos producto de entrevistas y cuestionarios.

Hace menos de una década, los estudios migratorios aseguraban que el teléfono era el primer artefacto tecnológico utilizado por los migrantes para contactarse con sus familias ${ }^{36}$. El segundo artefacto empleado era Internet. Sin embargo, a partir del relevamiento realizado por medio de entrevistas en profundidad y cuestionarios advertimos que las redes sociales virtuales, especialmente los programas como Facebook y Skype, resultan ser los medios más utilizados por los migrantes para comunicarse con sus países de origen, ya que ambos permiten una comunicación asidua, con poco gasto de dinero -solo la conexión a Internet- y que admite la llagada inmediata de la voz y/o de la

\footnotetext{
${ }^{35}$ MELELLA, Cecilia, PERRET, Gimena. El uso de Internet en contextos migratorios. Una aproximación a su estudio.

${ }^{36}$ MEJÍA ESTEVEZ, op. cit., entre otros.
} 
imagen generando modalidades de contacto caracterizadas por la intensificación a la apelación emotiva, somática y pulsional ${ }^{37}$. En segundo lugar, los migrantes entrevistados admiten que el teléfono celular, los e-mails y el teléfono fijo perduran como medios usuales de contacto con sus redes sociales en origen. No obstante, hoy en día estas tecnologías coexisten con otros programas y/o dispositivos como Whatsapp -que además de permitir enviar mensajes de texto con un costo muy bajo, también habilita la comunicación telefónica- y Twitter -que sostiene una comunicación instantánea y de índole pública-. El 70\% de los entrevistados utiliza Facebook, seguido de Skype $(64,5)$, del teléfono celular $(45,8)$ y de los e-mails $(41,6)$ (Figura 1$)$.

En cuanto a los usos y los intereses que impulsan a los migrantes al empleo de las TIC, los testimonios de los entrevistados se concentran en el acceso a los medios de comunicación de su país de origen y a la búsqueda de información tanto de su tierra natal como de la nación de destino. El consumo de los medios de comunicación de masas posibilita el contacto con el universo simbólico del destino en el origen posibilitando que los migrantes no escindan afectiva ni comunicativamente el contacto con su tierra natal. Por consiguiente, la posibilidad de leer (en tiempo real) la prensa de sus países o la prensa de cada colectividad emitida en la sociedad receptora conectan aún más el aquí y el allá y fortalecen el paradigma del transnacionalismo ${ }^{38}$.

Por otra parte, para los migrantes internacionales el uso de estos dispositivos tecnológicos contribuye a forjar una sensación de cercanía y una dilación de la nostalgia y el sentimiento de pérdida. La mayoría de los entrevistados ha declarado que las TIC ayudan a sentirse más cerca y palian la angustia de estar lejos de su tierra y de sus seres queridos como lo demuestran los extractos de tres entrevistas representativas:

[Las TIC te ayudan a] estar enterado de lo que sucede en tu país de origen a tiempo real, quién ganó el campeonato de fútbol, por qué cuestión la gente se manifiesta en las calles o quien ganó un concurso televisivo. Siempre pienso en mi abuelo y qué distinta fue su situación, esas cartas eternas que tardaban meses en llegar. Ahora que lo pienso en el año 2004 escribí un par de cartas a mis amigos enviando fotos. No existía Facebook ni celulares con cámara de fotos. (Ahora) si no encuentro a personas en el chat, las busco por Skype y, sino, acudo al teléfono fijo. Verse y contarse cosas cotidianas te hacen sentir más cerca de tus seres queridos, es más, cuando los vuelves a ver, pasado un período considerado, te parece que no fue tanto tiempo el que pasó sin contacto físico (Entrevista ${ }^{\circ} 48$ ).

\footnotetext{
${ }^{37}$ VALDETTARO, Sandra. Comentarios sobre una experiencia de investigación sobre las estrategias discursivas de la prensa actual.

${ }^{38}$ APPADURAI, op. cit.; VERTOVEC, Steven. Transnational social formations: Towards conceptual cross-fertilization; PORTES, op. cit.
} 
[También han subrayado la importancia de estar conectado] (...) definitivamente, me ayuda a sentirme conectada con mi país y mis amigos, a no sentirme sola, y quiero saber si pasan nuevas cosas en mi país. Whatsapp/ Facebook (móvil o portátil) ayudan a estar conectado con la gente que se encuentra aquí. Es una forma muy fácil para poderse encontrar y quedar juntos (Entrevista $\mathrm{n}^{\circ} 43$ ).

(...) no hace falta hablar cada día con mis amigos y mi familia, a veces no tienes nada (nuevo) que contar y siguen su vida cotidiana. Pero si hay un problema o si te sientes solo/a, tienes la oportunidad de llamar y hablar con las personas que te quieren y te dicen que todo vaya bien (Entrevista $n^{\circ} 40$ ).

Asimismo, refuerza la preservación de su cultura e identidad "(...) las tecnologías me sirven para mantenerme en contacto con mi familia y amigos, no olvidar mi cultura, mi acento, noticias de mi tierra, política, economía, etcétera $(\ldots)^{\prime \prime}$ (Entrevista ${ }^{\circ} 33$ ). El uso de las redes sociales para la búsqueda de trabajo en el país receptor, así como la obtención de información para la realización de trámites burocráticos y/o migratorios también constituyen actividades que los migrantes realizan a través de las TIC (Figura 2).

\section{Figura 2 - Principales usos de las TIC por parte de los migrantes sudamericanos en la Argentina}

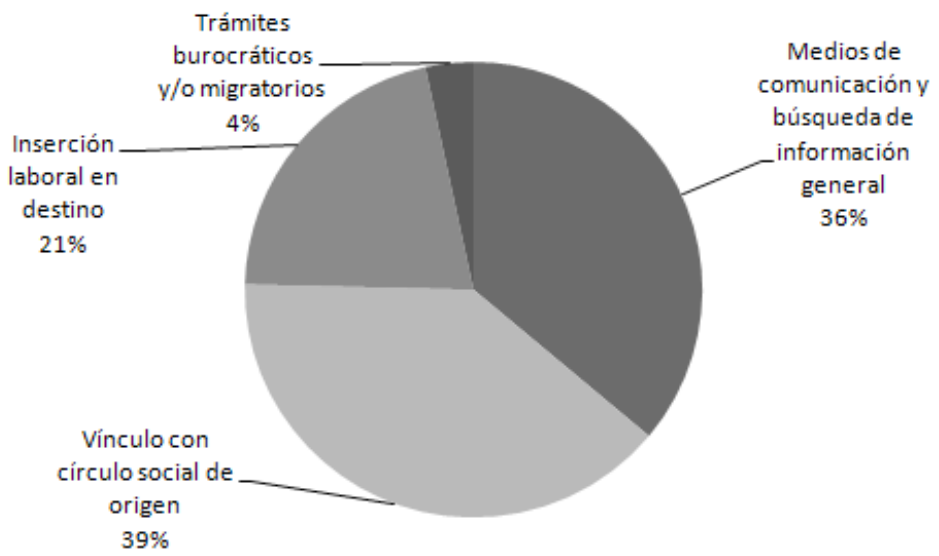

Fuente: Elaboración personal sobre la base de datos producto de entrevistas y cuestionarios.

Por último, dentro de las temáticas que más interés despiertan se encuentra la política y la economía en el origen y el destino; la información sobre derechos, en especial aquellos vinculados a la migración y al género, que han tenido en la Argentina de la última década una ampliación considerable ${ }^{39}$. Por otra parte, la

${ }^{39}$ Contemplamos que la siguiente normativa introduce pautas que abogan por la construcción de una sociedad que se encamina a la ampliación de derechos en un contexto intercultural en la Argentina: Decreto 1086/2005 "Hacia un Plan Nacional contra la discriminación": Documento diagnóstico y propositivo sobre discriminación; la Ley Nacional de Educación 26. 206 (2006): 
agenda cultural y la posibilidad de actividades recreativas que ofrece la sociedad de acogida representan otro tópico de beneficio para estos grupos migratorios, así como la información general sobre la Argentina (servicios de transporte, zonas turísticas, búsqueda de alojamiento, entre otras) y las noticias sobre su colectividad de origen en el destino (Figura 3).

\section{Figura 3 - Temáticas de interés para los migrantes sudamericanos en la Argentina}

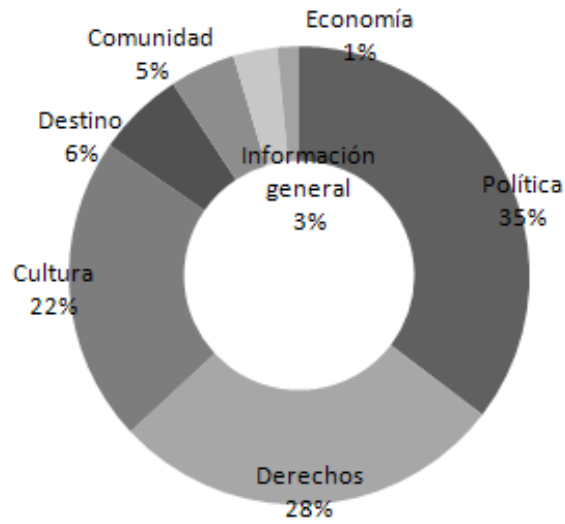

Fuente: Elaboración personal sobre la base de datos producto de entrevistas y cuestionarios.

\section{5 - Consideraciones finales}

En primer lugar, advertimos que el estudio de los medios de comunicación y especialmente del uso de las TIC adquiere mayor importancia debido a la trascendencia que ocupan en la vida de los migrantes y en el vivir transnacional que proponen las sociedades globales. En segundo lugar, en este artículo, hemos partido de la base de que la apropiación y el uso de las TIC resultan recursos estratégicos para los migrantes desde el plano colectivo (medios de comunicación comunitarios, portales y/o cuentas de Facebook de asociaciones de diversa índole) para el establecimiento de lazos de cohesión interna, medios informativos y solidarios, recursos de visibilidad y espacios para la conformación identitaria. En tercer término, hemos afirmado que también el uso de las TIC establece frondosas posibilidades desde el plano subjetivo, ya que abona el terreno para la configuración de las biografías migratorias desplegadas día a día. Los migrantes entrevistados han valorizado el uso de las TIC como herramientas y dispositivos que les permiten

Promoción de igualdad educativa en contextos de vulnerabilidad y educación Intercultural Bilingüe para pueblos originarios/indígenas; la Ley de servicios de comunicación audiovisual (2009): Radio y Tv para pueblos originarios y organizaciones son fines de lucro. Promueve la difusión de contenidos igualitarios basados en la diversidad; el Decreto Presidencial 1584/2010 por el cual el 12 de octubre cambió su nombre de "Día de la Raza" a "Día del respeto a la diversidad cultural" y la Ley 26.852 (2013): Día conmemorativo de la cultura afroargentina. 
situarse "más cerca" de sus redes sociales en origen y de esta manera cumplir aquello que Dana Diminescu denomina doble presencia migratoria (en origen y en destino). Además, destacan que el hecho de poder conectarse asiduamente y en tiempo real con sus seres queridos tiene el efecto de disminuir la nostalgia, entendida como nostós -la añoranza del regreso-. Asimismo, la vinculación con las TIC permite a los migrantes recrear su universo simbólico al estar informados a través de los medios de comunicación de origen o poder consumir los productos culturales, por ejemplo, en su propia lengua y con su bagaje identitario que, de alguna manera, genera una experiencia de proximidad.

En cuarto lugar, advertimos que las redes sociales virtuales como Facebook o los programas como Skype parecieran imponerse como las tecnologías preferidas para la comunicación con el origen, superando -o por lo menos disputando- el lugar que ocupaba el teléfono fijo. También resulta destacable que el espectro temático de interés tiene como eje la tríada compuesta por la política, la cultura y los derechos. No obstante, también los migrantes entrevistados han reconocido que el uso de las TIC (como Internet) antes de migrar les permitió realizar un reconocimiento a distancia de la sociedad de destino, además de la búsqueda de información y establecimiento de redes, acción que les brindó una mayor seguridad personal a la hora de migrar. En síntesis, si bien los estudios que vinculan las TIC y la migración se identifican por su carácter neófito todo el mundo, y aún más en la Argentina, resulta ser una línea de investigación que se impone en el campo migratorio. En un contexto migratorio sur-sur, el estudio sobre las TIC abre posibilidades para reflexionar sobre incipientes modos de socialización/ comunicación transnacional que, construidos sobre el espacio virtual, propician la conformación de narrativas y/o identidades migratorias (virtuales) bajo otra percepción del espacio-tiempo cercana a la circulación y la instantaneidad.

\section{Bibliografía}

APPADURAI, Arjun. La modernidad desbordada: Dimensiones culturales de la globalización. Buenos Aires: Fondo de Cultura Económica, 2001.

ARFUCH, Leonor (comp.). Identidades, sujetos y subjetividades. Buenos Aires: Prometeo, 2002.

ARFUCH, Leonor. El espacio biográfico: Dilemas de la subjetividad contemporánea.

Buenos Aires: Fondo de Cultura Económica, 2010.

BRUNEAU, Michel. Diaspora, transnational spaces and communities. In BAUBÖCK, Rainer; FAIST, Thomas (eds.). Diaspora and Transnationalism: Concepts, Theories and Methods. Amsterdam: University Press, 2010, p. 35-50.

CACHÓN RODRÍGUEZ, Lorenzo. La España inmigrante. Marco discriminatorio, mercado de trabajo y políticas de integración. Barcelona: Anthropos, 2009.

CARDON, Dominique. La innovación por el uso. Palabras en juego. Enfoques Multiculturales sobre las Sociedades de la Información. 2006. Disponible en: < http:// www.casanas.com.ar/artsAdj/Palabras_en_juego-221.pdf>. Consultado: 02.10.2015. 
CASTELLO STARKOFF, Paula; BURBANO, Mauricio. Migración ecuatoriana y uso de Nuevas Tecnologías de Información y Comunicación. Cartillas sobre la migración. Plan Migración, Comunicación y Desarrollo, Ecuador, n. 12. 2005. Disponible en: <http://www.edured.ec/web_html/documentos/Biblioteca_virtual/Migracion/ Migracion_ecuatoriana_y_uso_de_las_TIC > . Consultado: 12.02.2015.

CASTELO STARKOFF, Paula; RAMIREZ, Jacques. Migración ecuatoriana y uso de nuevas tecnologías de Información y Comunicación. Quito: Plan Migración, Comunicación y Desarrollo, 2006.

CASTELLS, Manuel. La Era de la Información: La Sociedad Red. Madrid: Alianza, 1995.

DIMINESCU, Dana. El migrante conectado. Por un manifiesto epistemológico. In HERNÁNDEZ, Valeria; MERA, Carolina; OTEIZA, Enrique; MEYER, Jean Baptiste (comps.). Circulación de saberes y movilidades internacionales: Perspectiva latinoamericana. Buenos Aires: Biblos, 2011, p. 201-216.

DIMINESCU, Dana. E-Disporas Atlas. Exploration and Cartography of Disaporas in Digital Networks. 2012. Disponible en: < http://www.inter-disciplinary.net/at-the-interface/ wp-content/uploads/2012/06/diminescudpaper.pdf>. Consultado: 12.06.2015.

GEORGIOU, Myria. Diasporic Media Across Europe: Multicultural Societies and the Universalism-Particularism Continuum. 2009. Disponible en: <http://eprints.Ise. ac.uk/25590/1/Diasporic_media_across_Europe_\%28LSERO_version\%29.pdf > . Consultado: 17.05.2015.

GÓMEZ-ESCALONILLA MORENO, Gloria; CAMPOS ZABALA, Marina. Espacios de solidaridad en Internet: Webs para inmigrantes. 2009. Disponible en: < http:// migraciones.ugr.es/images/seminarios/representacionmedios/2009Campos.pdf > . Consultado: 12.02.2015.

GONZÁlEZ CORTÉS, María Eugenia; ARRANQUERO CARRETERO, Alejandro. Empleo y usos de internet en las comunidades imaginadas: la red como herramienta neutralizadora de la distancia. 2006. Disponible en: < http://www.razonypalabra.org.mx/anteriores/ n49/bienal/Mesa\%209/CortesBarrenquero.pdf>. Consultado: 02.10.2015.

GONZÁLVEZ TORRALBO, Herminia. Comunicación y contacto transnacional: El uso de las nuevas tecnologías de información y comunicación en la constitución de redes migratorias. In REVILLA BLANCO, Marisa; GÓMEZ JOHNSON, Cristina (eds.). Caminos de ida y vuelta: redes, migración y desarrollo. Madrid: Catarata, 2012, p. 110-133.

GOUBE, Josephine. When immigration meets innovation: immigrants 2.0. Migrant's rights network. 2012. Disponible en: < http://www.migrantsrights.org.uk/migration-pulse/2012/ when-immigration-meets-innovation-immigrant-20>. Consultado: 02.10.2015.

HINE, Christine. Etnografía virtual. Barcelona: UOC, 2004.

KISSAU, Kathrin; HUNGER, Uwe. The internet as a means of studying transnationalism and diaspora. In BAUBÖCK Rainer; FAIST, Thomas (eds.). Diaspora and Transnationalism: Concepts, Theories and Methods. Amsterdam: University Press, 2010, p. 246-265.

MARTÍN-BARBERO, Jesús. De los medios a las mediaciones: Comunicación, cultura y hegemonía. Barcelona: Gustavo Gili Editores, 1991. 
MEJÍA ESTEVEZ, Silvia. Transnacionalismo a la ecuatoriana: migración, nostalgia y nuevas tecnologías. In HERRERA, Gioconda; CARRILLO, María Cristina; TORRES, Alicia (eds.). La migración ecuatoriana, transnacionalismo, redes e identidades. Quito: Flacso, 2005, p. 481-492.

MELELLA, Cecilia. La prensa migrante en la Argentina. Recurso de visibilidad y construcción de identidades: El caso del periódico Renacer, de y para la colectividad boliviana. Saarbrücken: Editorial Académica Española, 2012.

MELELLA, Cecilia. Apropiación y uso de las tecnologías de la información y de la comunicación (TIC) en la prensa migrante de las colectividades andinas en la Argentina. Revista Doxa Comunicación, v. 18, 2013, p. 141-157.

MELELLA, Cecilia. Migración y Tecnologías de la Información y de la Comunicación (TIC). La presencia de los periódicos de migrantes en Internet y los desafíos del análisis de las redes sociales virtuales. Cuadernos de H Ideas, v. 7, n. 7, 2013, p. 1-18.

MELELLA, Cecilia. Migración y tecnologías de la información y la comunicación (TIC). De la prensa gráfica a las redes virtuales como espacios de construcción identitaria de los migrantes de países andinos en la Argentina. Tesis de doctorado no publicada. Universidad de Buenos Aires, Facultad de Ciencias Sociales, Argentina, 2013.

MELELLA, Cecilia. MIGRANTES.COM. Las asociaciones de migrantes andinos en la Web. 2014. Disponible en: < http://redcomunicacion.org/> Consultado: 12.03.2015.

MELELLA, Cecilia. Migración y TIC: Identidades andinas en Facebook. La Trama de la comunicación, 20 (en prensa), 2016.

MELELLA, Cecilia; PERRET, Gimena. Tecnologías de la Información y de la Comunicación y estudios migratorios. Reflexiones sobre un campo de estudios incipiente. Ponencia presentada en VIII Jornadas de Sociología de la UNLP. 3 - 5 de diciembre, 2014. La Plata. Disponible en: < http://jornadassociologia.fahce.unlp.edu.ar/viiijornadas-2014/PONMesa44Melella.pdf/view>. Consultado: 02.10.2015.

MELELLA, Cecilia; PERRET, Gimena. El uso de Internet en contextos migratorios. Una aproximación a su estudio. Cuadernos de trabajo Seminario interno de metodología. Buenos Aires: Facultad de Filosofía y letras. Universidad de Buenos Aires, 2015.

PEÑARANDA CÓlERA, María Cristina. ¿Tecnologías que acercan distancias? Sobre los 'claroscuros' del estudios de la(s) tecnología(s) en los procesos migratorios transnacionales. In SANTAMARÍA, Enrique (ed.). Retos epistemológicos de las migraciones transnacionales. Barcelona: Antrophos, 2008, p. 135-167.

PERRET, Gimena. Nuevas tecnologías de la información, formas de organización migrante y lucha por derechos políticos de la migración chilena en Argentina. Ponencia presentada en VII Jornadas de Sociología, 24 - 25 de abril, 2012. Buenos Aires. Disponible en: <http://www.ungs.edu.ar/ms_ici/wp-content/uploads/2012/11/ Perret-PonenciaVIIJornadasSociologiaUNGS_GT1.pdf > . Consultado: 02.10.2015.

PORTES, Alejandro. Convergencias teóricas y evidencias empíricas en el estudio del transnacionalismo de los inmigrantes. Migración y desarrollo, n. 4, 2005, p. 2-19.

VALDETTARO, Sandra. Comentarios sobre una experiencia de investigación sobre las estrategias discursivas de la prensa actual. Ponencia presentada en Jornadas de Intercambio Académico: "Miradas discursivas sobre la prensa gráfica y electrónica". 
29 de abril de 2006. Buenos Aires. Disponible en: <http://jornadas2006.blogspot. com/> Consultado: 02.10.2015.

VASILACHIS DE GIALDINO, Irene. Estrategias cualitativas de investigación. Buenos Aires: Gedisa, 2007.

VERTOVEC, Steven. Transnational social formations: Towards conceptual cross-fertilization. 2001. Ponencia presentada en "Transnational Migration: Comparative Perspectives", 30 de junio - 1 de julio, 2001. Princeton University. Disponible en: <http://www. transcomm.ox.ac.uk/working\%20papers/Vertovec2.pdf $>$. Consultado: 02.10.2015.

WINOCUR, Rosalía. La apropiación de la computadora e Internet en los sectores populares urbanos. Revista Versión, n. 19, 2007, p. 191-216.

\section{Abstract \\ The use of information technology and communication (ICT) for the South American immigrants in Argentina and the shaping of transnational social networks}

The use of ICT implies a turning point for traditional migration processes because enable the uprooted migrant become a connected migrant. The ICT, especially the Internet, creates a lush universe of possibilities of connection and communication with the countries of origin, with the diaspora and with the community of arrival amending the construction of migrant subjectivity and form and transnational social networks. In this article, we plan to see the relevance of ICT, beyond the conception of tools and cultural artifacts of the public sphere, as devices that migrants turn to build as a referential space, which supports the conservation of regulars links, social networks at origin and mitigate or reconfigure the nostalgia of the absence and return. Through conducting interviews and questionnaires, We intend to study the means and technologies often used by individuals of these groups to communicate with their social networks of origen, the causes of their choice, their frequently uses and the subjects that arouse them their interest when resorting to these technological devices.

Keywords: South American migration, information technology and communication (ICT), appropriation and use, connected migrant, transnational social networks.

Recibido para publicación en 05.01.2016

Aceptado para publicación en 06.03.2016 Received for publication in January 05 ${ }^{\text {th }}, 2016$ Accepted for publication in March 06 ${ }^{\text {th }}, 2016$ 